\title{
Fibre Bragg grating inscription into a seven core fibre and its application as a vector bending sensor
}

Sahoo, Namita, Bao, Weijia, Liu, Shen, Tan, Yidong, Zhou, Kaiming, et al.

Namita Sahoo, Weijia Bao, Shen Liu, Yidong Tan, Kaiming Zhou, Lin Zhang, "Fibre Bragg grating inscription into a seven core fibre and its application as a vector bending sensor," Proc. SPIE 11772, Optical Sensors 2021, 1177200 (18 April 2021); doi: 10.1117/12.2589063

SPIE. Event: SPIE Optics + Optoelectronics, 2021, Online Only 


\title{
Fibre Bragg grating inscription into a seven core fibre and its application as a vector bending sensor
}

\author{
Namita Sahoo, ${ }^{\mathrm{a},}$, Weijia Bao ${ }^{\mathrm{b}}$, Shen Liu ${ }^{\mathrm{b}}$, Yidong Tan ${ }^{\mathrm{c}}$, Kaiming Zhou ${ }^{\mathrm{a}}$, Lin Zhang ${ }^{\mathrm{a}}$ \\ a Aston Institute of Photonic Technologies, Aston University, Birmingham, United Kingdom \\ ${ }^{\mathrm{b}}$ Guangdong and Hong Kong Joint Research Centre for Optical Fibre Sensors, Shenzhen University, \\ China \\ ${ }^{c}$ Department of Precision Instrument, Tsinghua University, Beijing, China
}

\begin{abstract}
This paper demonstrates a highly effective method for fibre Bragg grating (FBG) inscription into a seven core fibre (7CF) and its application as an effective vector bending sensor. The development of multicore fibres (MCFs) and enabling the fabrication of FBGs into them result in a solution for multi-parameter measurements such as temperature/strain/bending/twist. The FBG in the central core of 7CF is on the neutral axis and therefore it is sensitive only to thermal and insensitive to deformational change, whereas the FBGs in the none-central cores that are evenly distributed over the 7CF cross-section can facilitate the measurement of structure deformation, such as bending, loading and twist. Furthermore, the FBGs in different cores respond to the physical perturbations differently in various orientations, offering vector sensing to measure both amplitude and direction of the structure change. The 7CF-FBG sensors are highly applicable for mechanical structures and flexible medical instruments.
\end{abstract}

Keywords: Fibre Bragg grating, multicore fibre, curvature sensing, twist sensing, vector sensors

\section{INTRODUCTION}

A fibre Bragg grating (FBG) is formed by a periodic perturbation to the refractive index within the core of a single mode fibre, generating a reflection resonance in spectrum. FBGs can be inscribed with irradiance from a frequency-doubled Argon ion laser at wavelength of $244 \mathrm{~nm}$ [1-4] and they have been used for sensing of different parameters such as temperature, strain, bending and twist and utilized for structural health monitoring and medical devices [5-8]. These FBG optical sensors are superior due to their high thermal stability, chemical inertness and immune to electromagnetic interference.

Multicore fibre (MCF) development, a recent advancement of fibre optical technology driven by huge demand for transmission capacity of a single fibre and long-haul communication with dense space-division multiplication, was reported [9-12]. In addition, key applications utilizing MCF such as fibre laser [13, 14] and fibre optical sensors [15, 16], were immediately followed. For these applications, existing device such as fibre gratings and optical couplers may be useable but requiring multiple splitting and combining between single core and multicore fibre. New version of these devices in MCFs will make the system more elegant. Furthermore, FBGs fabricated into the MCFs can broaden their capabilities in regarding to multi-parameter sensing of twist, bending, loading and temperature.

Here we report the fabrication of FBGs in a seven-core fibre (7CF) with the central one used as a temperature reference and those in the outer cores are demonstrated for two dimensional vector bending and twist sensing.

\section{FBG FABRICATION IN 7CF BY SELECTIVE CORE INSCRIPTION}

The 7CF (SM-7C1500) was bought from Fibercore with each core and cladding diameters of $6.1 \mu \mathrm{m}$ and $125 \mu \mathrm{m}$ respectively. The cores are equally spaced apart with a $35 \mu \mathrm{m}$ distance from each other, as seen through optical fibre cross section with an optical microscope (ZEISS Axioscope), as shown in Fig. 1(a). In order to modulate the core refractive index to generate FBG structure, the 7CF was enhanced for the UV photosensitivity by hydrogen loading technique, with which the fibre was kept in a hydrogen loader under a constant pressure of 150 bar and at a temperature of $80{ }^{\circ} \mathrm{C}$ for 48 hours.

*E-mail: $\underline{\text { sahoon@aston.ac.uk }}$

Optical Sensors 2021, edited by Francesco Baldini, Jiri Homola, Robert A. Lieberman, Proc. of SPIE

Vol. 11772, $1177200 \cdot$ @ 2021 SPIE · CCC code: 0277-786X/21/\$21 · doi: 10.1117/12.2589063 


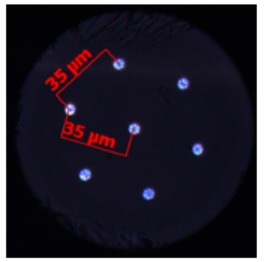

(a)

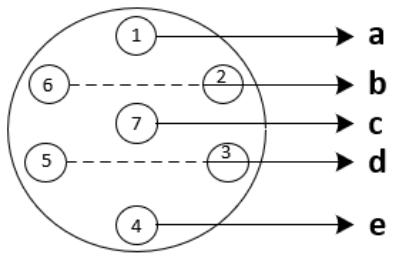

(b)

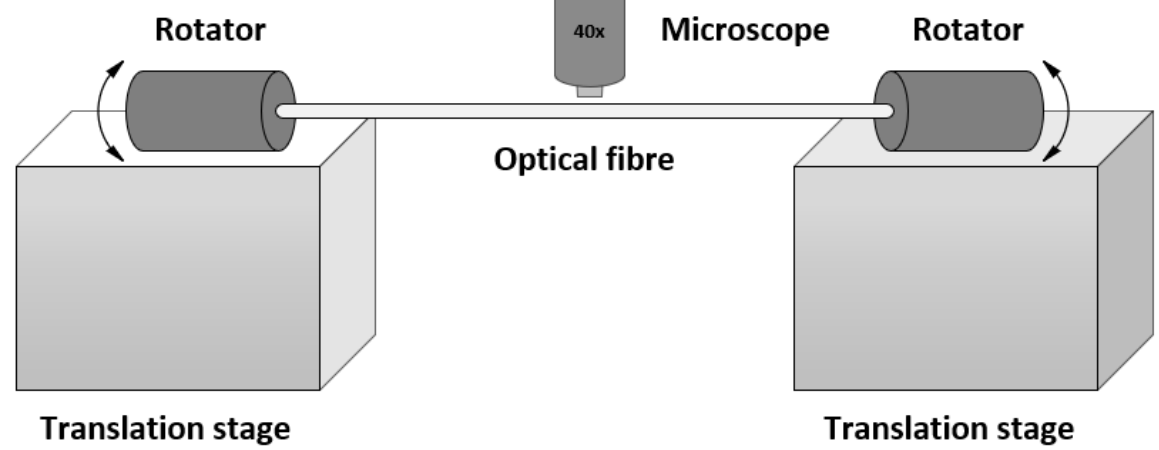

(c)

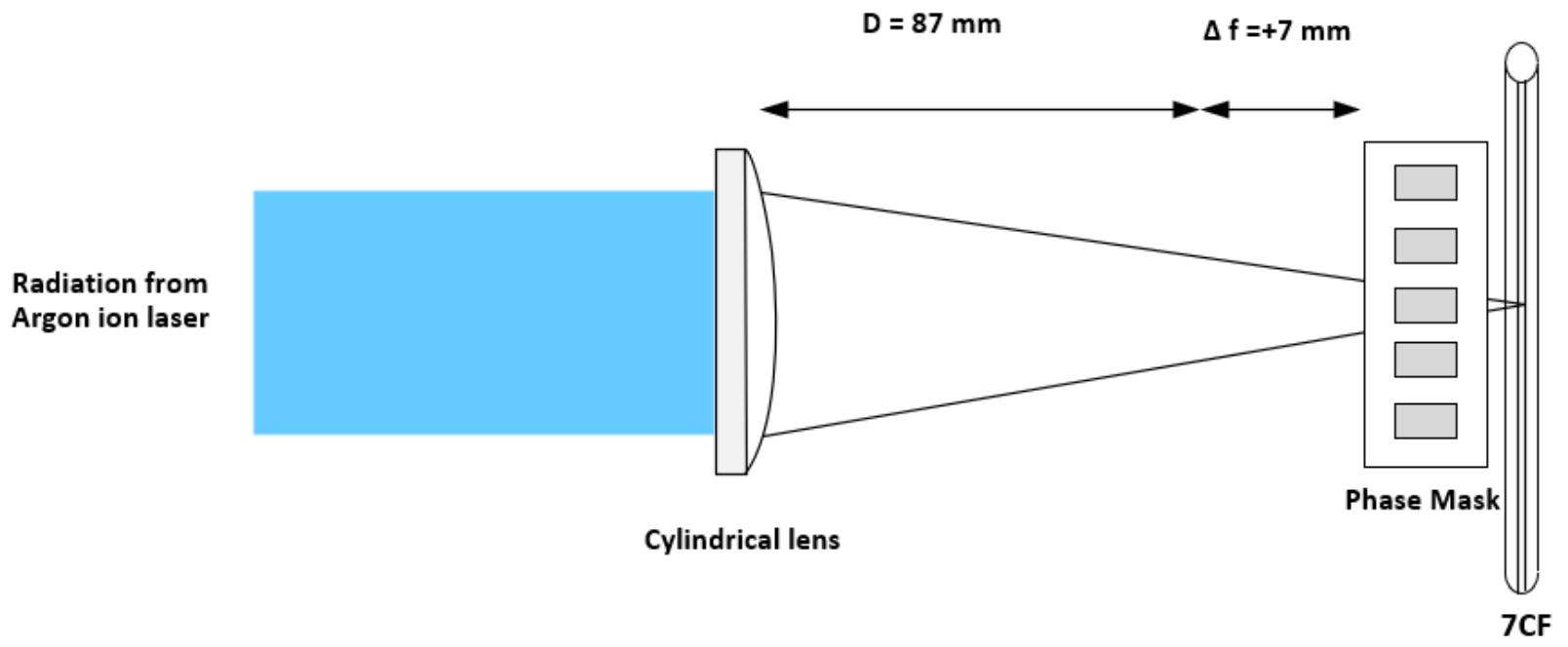

(d)

Figure 1.(a) The cross-section image of the 7CF taken under a 40x microscope; (b) the 5 positions (a, b, c, d, e) marked for the 7CF; (c) the set-up to mark the core position of 7CF; (d) UV inscription scheme for FBG fabrication in 7CF.

The fabrication technique used for FBG inscription into 7CF comprises phase mask method by focussing an Argon ion laser beam (Coherent Innova 300) into the fibre core using a cylindrical lens (Fig.1d). The FBG inscription is performed with a laser power output of $100 \mathrm{~mW}$ and a scanning velocity of $0.05 \mathrm{~mm} / \mathrm{s}$ for a length of $15 \mathrm{~mm}$. The selective inscription is dependent on the core marking before the inscription. As shown in Fig. 1(c), the 7CF, clamped with two fibre rotators, is positioned between two 3D stages with a $20 \mathrm{~cm}$ separation between them. A microscope objective with a magnification of 40x is used for observation of the core position. The rotators can be adjusted according to the requirement of the core alignment within the fibre. Once the position and orientation of the core are aligned, the fibre is fixed to a firm position for the UV inscription. Figure 1(b) shows the 5 core positions are identified and labelled as (a, b, c, d, e), where for (a), (c) 
and (e) are the top, central and bottom cores respectively, whereas the two cores ( $2 \& 6$ and $3 \& 5)$ in a same plane are marked as (b) and (d). It is intended to inscribe FBGs into all the cores of the 7CF with the centre wavelength around 1550 $\mathrm{nm}$. After the inscription, the FBG spectra of all cores are measured using a butt-coupling method, as shown in Fig. 2. The measurement method comprises a 3-dB coupler to launch the light from a broadband source (BBS 1550), a splicer with precise and visualisable adjustment of $\mathrm{X}-\mathrm{Y}$ axis used for coupling light into different cores and an optical spectrum analyzer (OSA YOKOGAWA) with a resolution of $0.05 \mathrm{~nm}$.

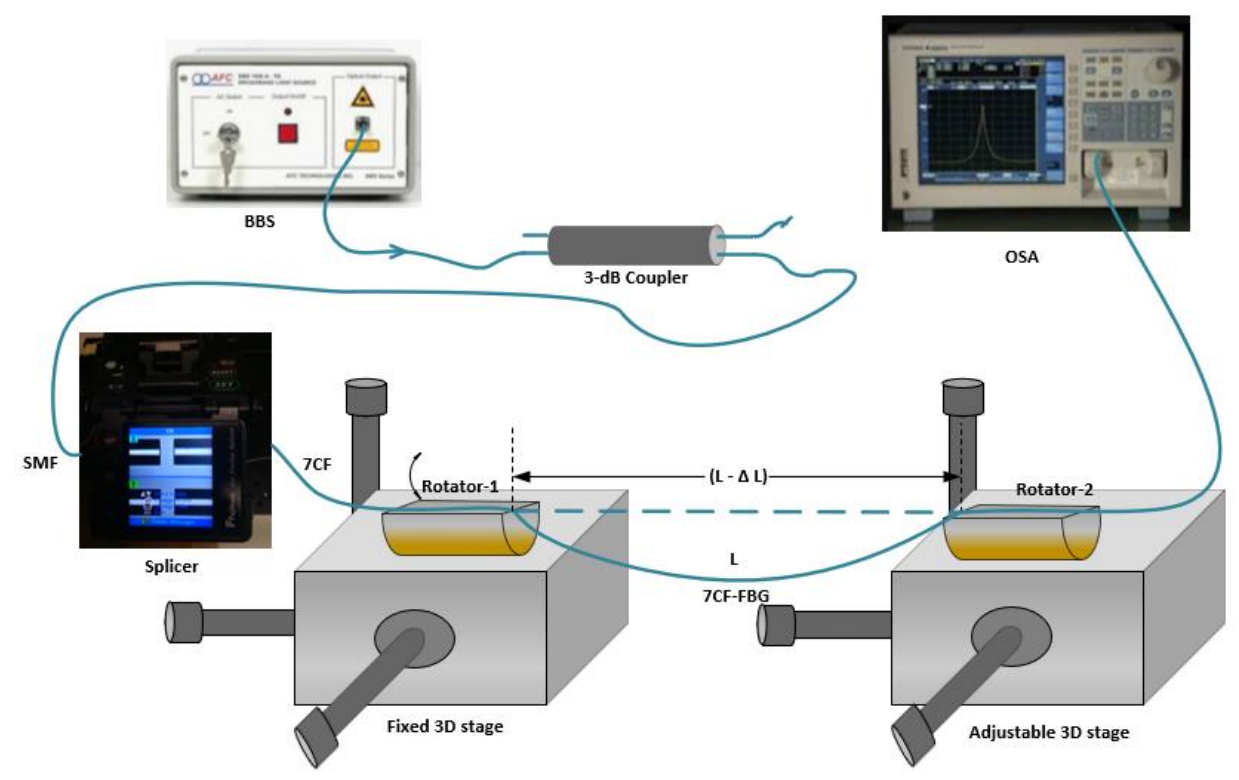

Figure 2. Schematic diagram of the measurement setup for 7CF FBG spectral characterisation and vector bending sensing.

Figure 3 shows the transmission spectra of FBGs inscribed into the central core and $3^{\text {rd }}$ and $6^{\text {th }}$ cores respectively. It is seen that the FBG resonances of these three cores are shifted to longer wavelengths ranging from $1555.7 \mathrm{~nm}$ to $1556.5 \mathrm{~nm}$. The maximum resonance (Bragg reflection) achieved for the central core is the strongest reaching $27 \mathrm{~dB}$, whereas for other two cores ( $3 \mathrm{rd} \& 6 \mathrm{th}$ ), it is approximately $15 \mathrm{~dB}$. The variation in resonance wavelength and strength may be due to the alignment and focusing condition and inscription efficiency. In principle, if the alignment and the UV beam focusing condition are precisely controlled, FBGs can be written into all cores with similar wavelength and strength. Although here we only show spectra of the FBGs in three cores, there are several samples with Bragg resonance shown for all seven cores.

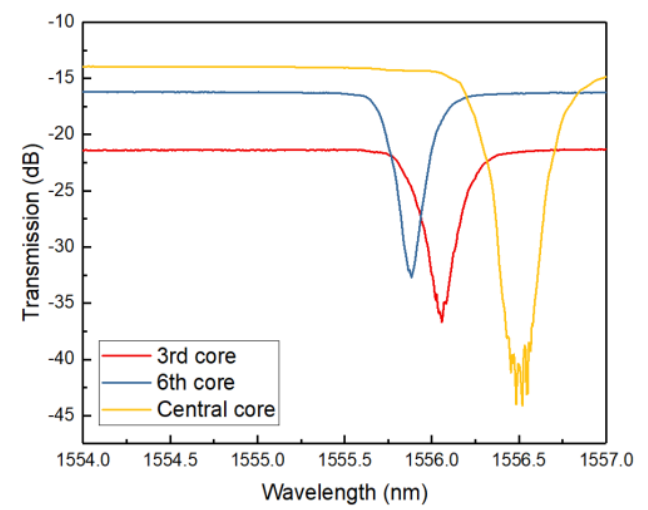

Figure 3. Transmission spectra for FBGs inscribed into $3^{\text {rd }}, 6^{\text {th }}$ and the central core of the $7 \mathrm{CF}$. 


\section{7CF-FBG BENDING SENSING UNDER TWIST}

Due to the FBGs are in the cores, distributed at different locations in $7 \mathrm{CF}$, vector sensing is possible as the FBG spectral responses of different cores will provide both direction and amplitude of the structure change, for example under bending and twisting. We may explain the vector sensing of 7CF-FBG corresponding to the bending in the orthogonal directions ( $\mathrm{x}$ and $\mathrm{y}$ ) as depicted in Fig. 4(a). The angle $\theta$ is formed between $\mathrm{y}$-axis (for $\theta=0^{\circ}$ ) and the distance $\mathrm{d}$ between central core and an outer core. Fig. 4(b) shows the curvature at $\theta=270^{\circ}$ and the propagating light is bent along the arc of a circle. Mathematically the change in bending curvature can be expressed as $\sin \left(\frac{L C}{2}\right)=\frac{(L-\Delta L)}{2} C$. Here, $\mathrm{C}$ is the radius of curvature $(1 / \mathrm{R})$ and can be calculated for different bending position of fibre [17]. $L$ is the initial distance between two fixed ends of the fibre, $\Delta L$ is the shift in distance of the 3D stage (Fig. 2). The bending is applied along the two-dimensional plane of the fibre.

For the twist and bending sensing measurement, the 7CF-FBG was placed between two 3D translation stages with a distance of $\mathrm{L}=12.5 \mathrm{~cm}$. The twist response is studied while one end of the fibre is fixed and the other side of the fibre is rotated at different angles of $0^{\circ}, 90^{\circ}, 180^{\circ}$ and $270^{\circ}$ respectively. The bending measurement is done with the inward movement of one 3D stage to introduce curvature to the fibre (shown in Fig. 2), and the maximum movement is $10 \mathrm{~mm}$. The spectrum for each bending is recorded on to the optical spectrum analyser. We clearly see the 7CF-FBG responds to the bending and the twisting, showing not just the spectrum shifting but also the shift direction according to the FBG in which core.

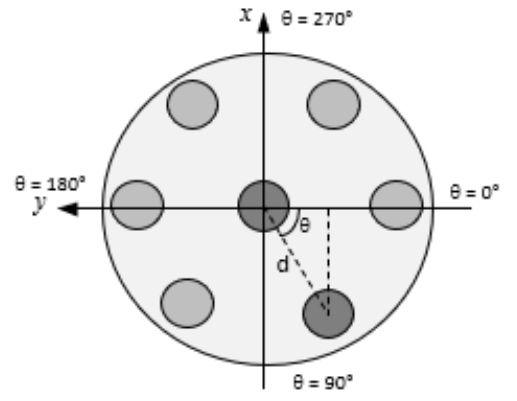

(a)

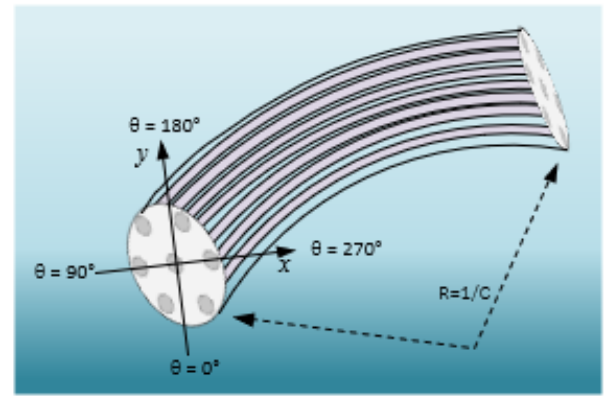

(b)

Figure 4. Schematic diagram for vector sensing mechanism [17].
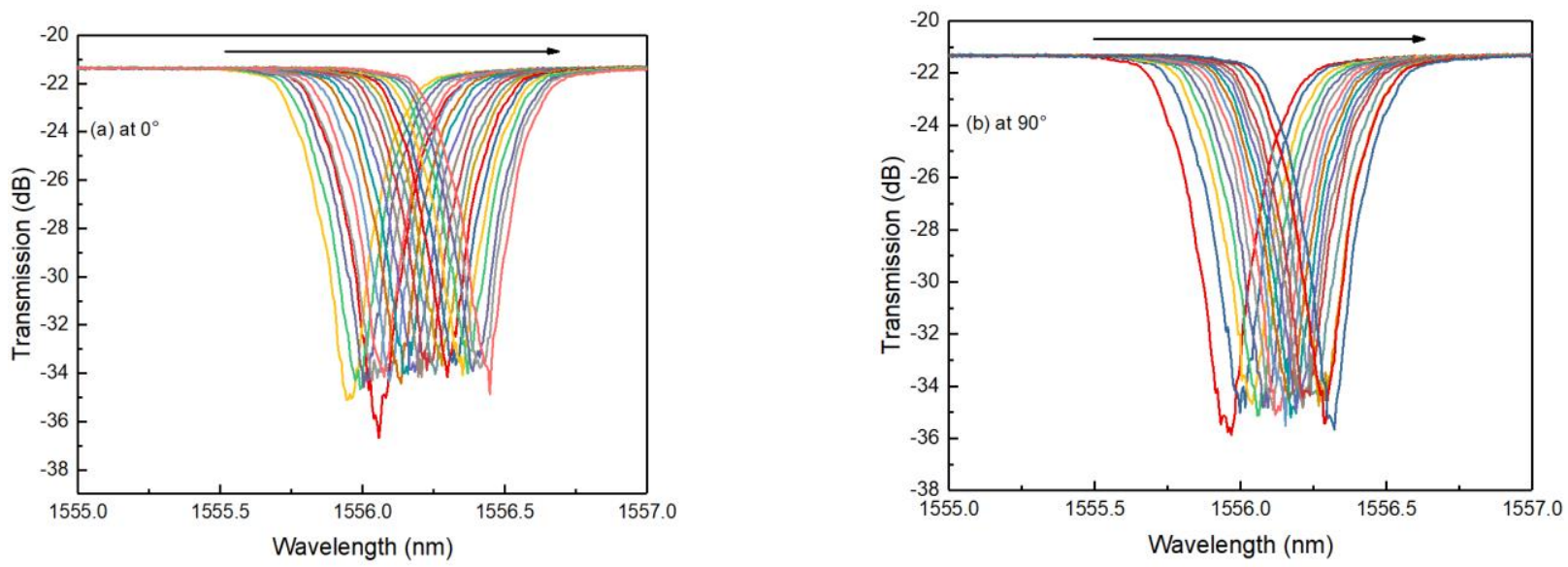

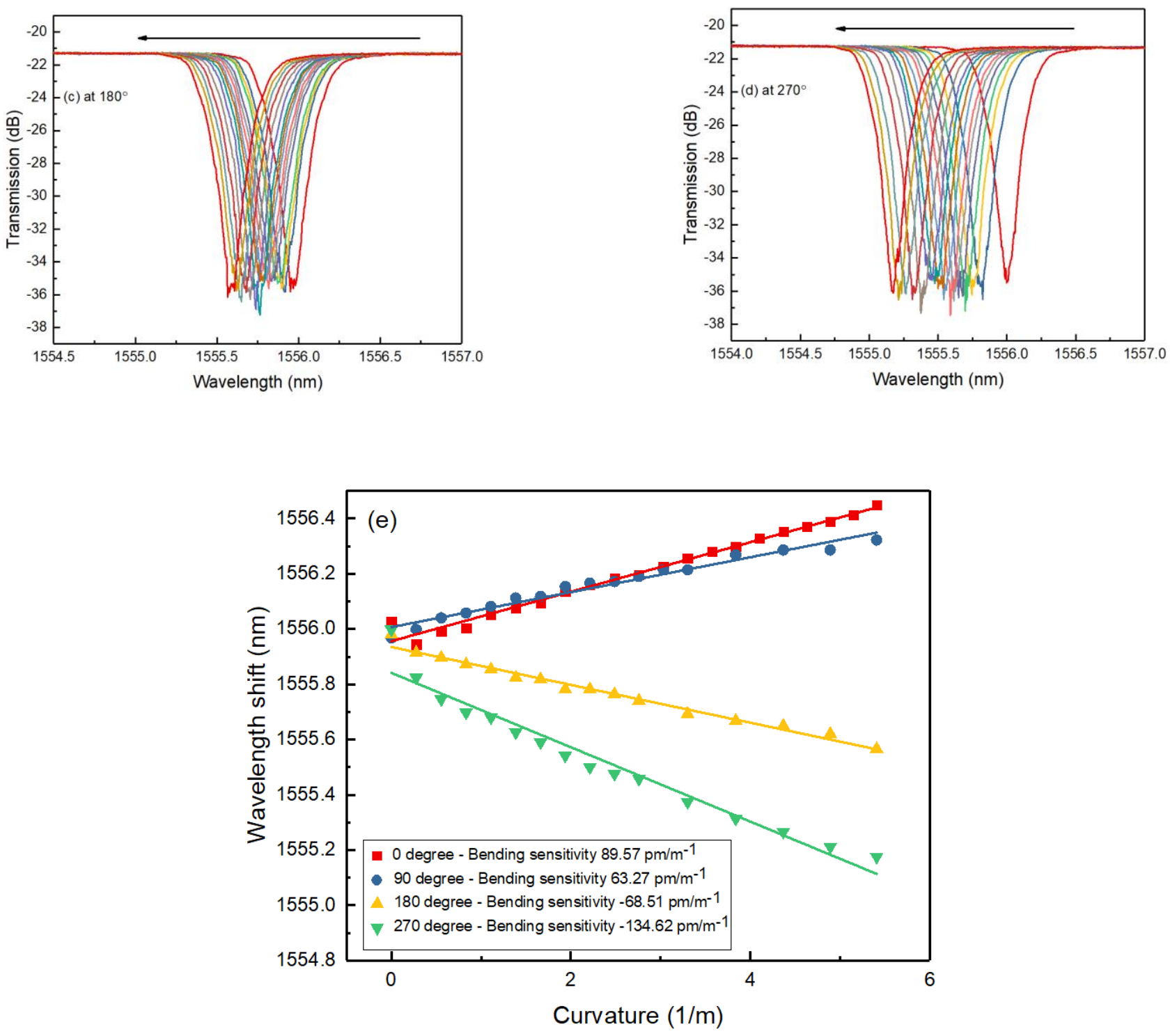

Figure 5. The $3^{\text {rd }}$ core FBG spectral responses when the fibre subjected to the bending under four twist angles: (a) $0^{\circ}$; (b) $90^{\circ}$; (c) $180^{\circ}$; (d) $270^{\circ}$. (e) Plotted bending sensitivities for the $3^{\text {rd }}$ core FBG, clearly showing the changes in curvature and in direction.

Figure 5 (a-d) display the spectral responses of the FBG inscribed in the $3^{\text {rd }}$ core when the fibre subjected to the bending and twisting at four different angles. From Fig. $5(\mathrm{a}, \mathrm{b})$ we can clearly see when the 7CF-FBG fibre is twisted at $0^{\circ}$ and $90^{\circ}$, the increase in bending applied to the fibre induces the Bragg resonance shifts towards the longer wavelength (redshift), whereas when the fibre is twisted at $180^{\circ}$ and $270^{\circ}$, results in Bragg resonance moves towards to shorter wavelength (blue-shift). We plotted the bending sensitivities at these four bending/twisting condition in Fig. 5(e), the two plots for $0^{\circ}$ and $90^{\circ}$ exhibit the upward slope with curvature responsivity of $89.57 \mathrm{pm} / \mathrm{m}^{-1}, 63.27 \mathrm{pm} / \mathrm{m}^{-1}$, respectively, and the two plots for $180^{\circ}$ and $270^{\circ}$ show downward slope with the curvature responsivity of $-68.51 \mathrm{pm} / \mathrm{m}^{-1}$ and $-134.62 \mathrm{pm} / \mathrm{m}^{-1}$, respectively. From the results we can see some inconsistence trends in curvature responsivity. For example, the curvature responsivity at $270^{\circ}$ is larger than at $180^{\circ}$ as expected, however, the results for $0^{\circ}$ and $90^{\circ}$ are irregular because the two plots crossover. This could be caused by the precision of the alignment and how accurate the bending was applied to the 7CF-FBG. We will evaluate more carefully in future experiment to improve the consistence in the results. 

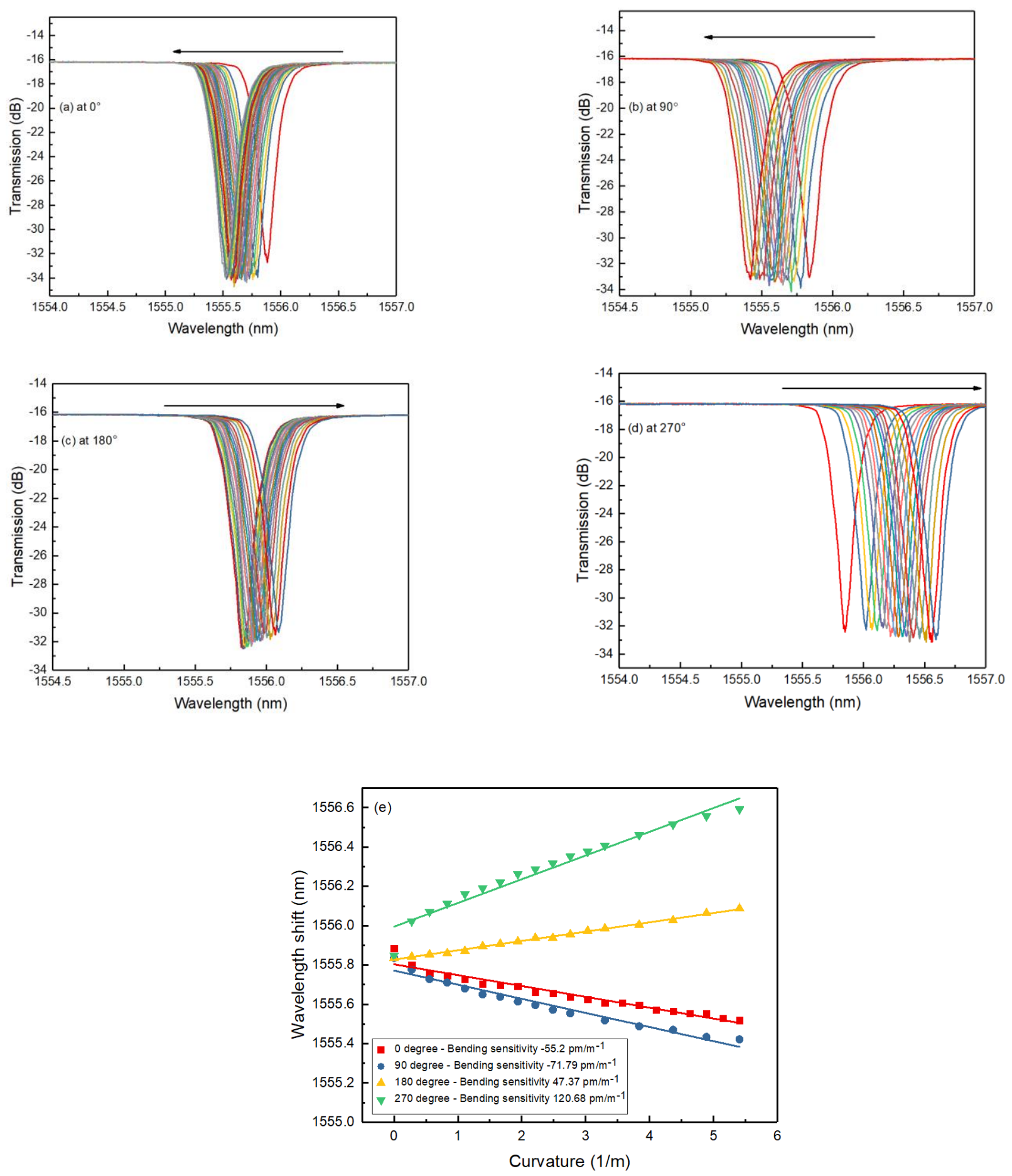

Figure 6 . The $6^{\text {th }}$ core FBG spectral responses when the fibre subjected to the bending under four twist angles: (a) $0^{\circ}$; (b) $90^{\circ}$; (c) $180^{\circ}$; (d) $270^{\circ}$. (e) Plotted bending sensitivities for the $6^{\text {th }}$ core FBG, clearly showing the changes in curvature and in direction.

To investigate the full bending/twisting capability of the 7CF-FBG, we also evaluated the FBG spectral change of the $6^{\text {th }}$ core, which is in the opposite position as the $3^{\text {rd }}$ core. Figure 6 (a-d) show the spectral evolution of the FBG inscribed in the $6^{\text {th }}$ core when the fibre subjected to the bending and twisting at $0^{\circ}, 90^{\circ}, 180^{\circ}$ and $270^{\circ}$. As the $6^{\text {th }}$ core is opposite to the $3^{\text {rd }}$ core, we expect the curvature induced Bragg resonance shifts will show opposite trends to the $3^{\text {rd }}$ core. From Fig. 6 
(a, b) we can clearly see when the fibre is twisted at $0^{\circ}$ and $90^{\circ}$, the increase in bending applied to the fibre induces the Bragg resonance blue-shifts, whereas when the fibre is twisted at $180^{\circ}$ and $270^{\circ}$, results in Bragg resonance of red-shifts. The bending sensitivity trends at the four twist angles are plotted in Fig. 6(e), showing the values of $-55.2 \mathrm{pm} / \mathrm{m}^{-1}$ and $71.79 \mathrm{pm} / \mathrm{m}^{-1}$ at $0^{\circ}$ and $90^{\circ}$, and $47.37 \mathrm{pm} / \mathrm{m}^{-1}$ and $120.68 \mathrm{pm} / \mathrm{m}^{-1}$ at $180^{\circ}$ and $270^{\circ}$, respectively. In an ideal model, as the $3^{\text {rd }}$ core and $6^{\text {th }}$ core are opposite and symmetrically distributed in the $7 \mathrm{CF}$, their spectral response to the bending under the same twist angle should be identical in sensitivity of amplitude and opposite in movement direction. Our experimental results of the $3^{\text {rd }}$ and $6^{\text {th }}$ core clearly show the opposite movements but with some discrepancy in bending sensitivity amplitudes due to errors in the fibre alignment and the applied curvature to the fibre.

As mentioned in the introduction, one of added unique advantages of vector sensing using the 7CF is that the central core will only be sensitive to the temperature and immune to the structure deformation caused by bending, loading or twisting. To prove this merit, we have also measured the spectral evolution of the central core labelled as 7 , and the results of bending response of the $7^{\text {th }}$ core FBG are given in Fig. 7. In comparison with the $3^{\text {rd }}$ and $6^{\text {th }}$ core, the Bragg resonance of the central core hardly moved (Fig. 7a). The wavelength shift against the curvature for the central core is plotted in Fig. 7(b), as shown the shift is so small as the maximum curvature caused resonance shift is less than $0.1 \mathrm{~nm}$, and the slope is only $-8.83 \mathrm{pm} / \mathrm{m}^{-1}$, which is about one order of magnitude lower than that of the $3^{\text {rd }}$ and $6^{\text {th }}$ core FBGs. This evidently indicates that the central core can be used as the reference to eliminate the temperature effect on the 7CF-FBG as structure monitoring sensor.
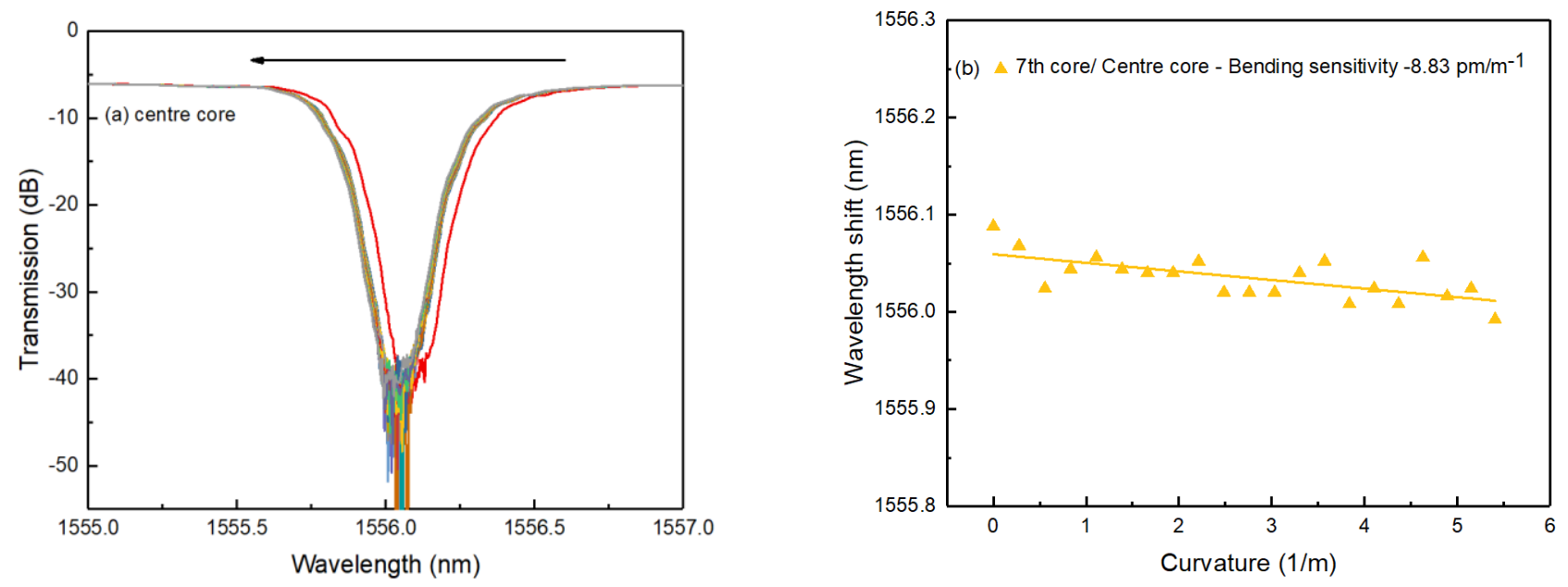

Figure 7 (a) The spectra of the central core FBG under bending change; (b) Wavelgth shift plot against the curvature for the central core.

\section{CONCLUSIONS}

We have demonstrated an effective fabrication technique to UV inscribed FBG structures to all 7 cores in a 7CF and the Bragg resonance can be as strong as $27 \mathrm{~dB}$. Due to the alignment condition and the fluctuation of the UV power, the FBGs in the distributed cores show some difference in Bragg resonance wavelength and strength. More precise alignment and control will reduce the discrepancy and generate more uniform FBGs in the 7 cores. We have also investigated the vector bending responses of the FBGs inscribed in the two oppositely situated cores under four twisting angles $\left(0^{\circ}, 90^{\circ}, 180^{\circ}\right.$ and $270^{\circ}$ ). The results clearly demonstrated the capability of such a 7CF-FBG as a vector sensor because their resonances not just respond to the curvature amplitude but also direction. In addition, we have also evaluated the central core as temperature reference function and the Bragg resonance of the central core showed insignificant response to the bending and twisting.

\section{REFERENCES}

Hill, K.O., et al., PHOTOSENSITIVITY IN OPTICAL FIBER WAVEGUIDES - APPLICATION TO REFLECTION FILTER FABRICATION. Applied Physics Letters, 1978. 32(10): p. 647-649. 
[2] Patrick, H. and S.L. Gilbert, Growth of Bragg gratings produced by continuous-wave ultraviolet light in optical fiber. Optics Letters, 1993. 18(18): p. 1484-1486.

[3] Erdogan, T. and V. Mizrahi, Characterization of UV-induced birefringence in photosensitive Ge-doped silica optical fibers. Journal of the Optical Society of America B, 1994. 11(10): p. 2100-2105.

[4] Poumellec, B., et al., UV induced densification during Bragg grating inscription in Ge:SiO2 preforms. Optical Materials, 1995. 4(4): p. 441-449.

[5] Kinet, D., et al., Fiber Bragg Grating Sensors toward Structural Health Monitoring in Composite Materials: Challenges and Solutions. Sensors, 2014. 14(4): p. 7394-7419.

[6] Westbrook, P.S., et al., Continuous Multicore Optical Fiber Grating Arrays for Distributed Sensing Applications. Journal of Lightwave Technology, 2017. 35(6): p. 1248-1252.

[7] Jantzen, S.L., et al., Individual inscription of spectrally multiplexed Bragg gratings in optical multicore fibers using small spot direct UV writing. Optics Express, 2020. 28(14): p. 21300-21309.

[8] Ye, X.W., Y.H. Su, and J.P. Han, Structural Health Monitoring of Civil Infrastructure Using Optical Fiber Sensing Technology: A Comprehensive Review. The Scientific World Journal, 2014. 2014: p. 652329.

[9] Mizuno, T., et al., Long-Haul Dense Space-Division Multiplexed Transmission Over Low-Crosstalk Heterogeneous 32-Core Transmission Line Using a Partial Recirculating Loop System. Journal of Lightwave Technology, 2017. 35(3): p. 488-498.

[10] Richardson, D.J., J.M. Fini, and L.E. Nelson, Space-division multiplexing in optical fibres. Nature Photonics, 2013. 7(5): p. 354-362.

[11] Saitoh, K. and S. Matsuo, Multicore Fiber Technology. Journal of Lightwave Technology, 2016. 34(1): p. 55-66.

[12] Essiambre, R., et al., Breakthroughs in Photonics 2012: Space-Division Multiplexing in Multimode and Multicore Fibers for High-Capacity Optical Communication. IEEE Photonics Journal, 2013. 5(2): p. 0701307-0701307.

[13] Anderson, J., et al. Multi-Core Fiber Lasers. in Frontiers in Optics 2015. 2015. San Jose, California: Optical Society of America.

[14] Kochanowicz, M., et al., Beam Quality of Multicore Fibre Lasers. Acta Physica Polonica A, 2010. 118.

[15] Silva-López, M., et al., Transverse load and orientation measurement with multicore fiber Bragg gratings. Applied Optics, 2005. 44(32): p. 6890-6897.

[16] Khan, F., et al., Curvature, twist and pose measurements using fiber Bragg gratings in multi-core fiber: A comparative study between helical and straight core fibers. Sensors and Actuators A: Physical, 2021. 317: p. 112442.

[17] Zhang, H., et al., Fiber Bragg gratings in heterogeneous multicore fiber for directional bending sensing. Journal of Optics, 2016. 18: p. 085705. 